\title{
Integración y comunicación en proyectos de investigación en el área de las ciencias sociales y la cultura
}

\author{
GRUPO DE TRABAJO DE LA RED \\ (José Amozurrutia) \\ Consejo Nacional para la Cultura y las Artes
}

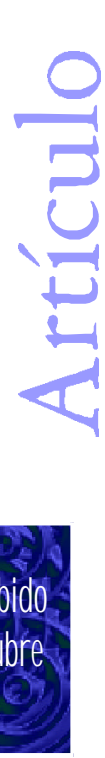

\begin{abstract}
RESUMEN
Este trabajo presenta los elementos técnicos y conceptuales que se toman en cuenta, para la conformación de una red de investigadores del área de las ciencias sociales y la cultura.

Como primer propuesta, se consideran aquellas investigaciones que requieren el uso de herramientas de cómputo para su desarrollo y presentación, por lo que los puntos centrales de la red son el uso del cómputo como herramienta vital, el manejo de la información y el uso de otros recursos que propicien el desarrollo autónomo de las investigaciones.

\section{ABSTRACT}

Thepaper presents the technical and conceptual elements which are taken into account when forming a researchers network in the area of social sciences and culture. A first proposal considers research that require the use of computer tools for their development and presentation. Thus, the central point of the network is the use of the computer as a vital tool, information handling and the use of resources which foster the autonomous development of research.
\end{abstract}

\section{INTRODUCCIÓN}

F l desarrollo de proyectos de investigación en las ciencias sociales y en la cul$\perp$ tura, con base en las herramientas de cómputo es una prioridad incuestionable hoy en día. Los múltiples vínculos, entre los proyectos de investigación, comprendidos desde su gestación hasta los frutos de sus primeras estaciones, deberían de existir al margen de las políticas sociales y culturales, y deben estar en empatía con los recursos tecnológicos cada vez más poderosos y al alcance de un creciente número de personas. La fuerza de dichos vínculos abrevia la vitalidad de los contactos directos.

Sin embargo, la realidad de muchos investigadores de las ciencias sociales y de la cultura en nuestro México, no en pocas ocasiones heterogénea, compleja y paradójica, nos enfrenta a un gran reto para pensar en la construcción de una "red de investigadores" que no sólo compartan títulos de proyectos, direcciones electrónicas y correos de sus sentires, sino una red de contactos que intercambie información relativa al estado de avance de los proyectos, a sus semillas, frutos y 
de especial manera, una red con espacios de intercomunicación, creación y recreación de la información, dentro de un contexto de amplio acceso, gran compartibilidad y uso de recursos al alcance del mayor número de investigadores y creadores.

La construcción de una red con tales características es un proyecto compartido por varios colegas y amigos deseosos de participar en la transformación de las "formas de relación y uso de los recursos" que ofrece la tecnología de las comunicaciones y el cómputo de hoy.

En estas líneas se sintetizan algunos de los elementos técnicos y conceptuales que ya se están conformando y construyendo para crear el componente en el Web de la red de investigadores y creadores en el vasto universo de las ciencias sociales y de la cultura en general, síntesis y conjunción de experiencias e ideas de algunos de sus gestores e integrantes. ${ }^{1}$ En este marco, el trabajo analiza el uso de elementos creativos para la intercomunicación entre los participantes de la red: investigadores y creadores, así como de la información compartible y la comunidad creciente en el ciberespacio.

\section{ASPECTOS GENERALES DE LA RED}

El concepto fundamental que conforma el componente en el Web de la red, se circunscribe a la interacción de dos esferas: una orientada al establecimiento de una estructura de contenidos, basada en formatos comunes y una madeja de hipertextos; y la esfera de creación, alimentada por las propuestas gráficas libres, con ligas abiertas para establecer los vínculos entre los contenidos, las personas y el entorno digital.

Los ejes centrales de nuestra propuesta son los siguientes:

1. El uso del cómputo como herramienta vital y autónoma para la construcción de información compartible y para la intercomunicación entre los integrantes de la red y la comunidad en general.

2. El manejo de la información con un mínimo de elementos para su organización y un máximo de elementos para su distribución y difusión.

3. El uso de recursos idóneos que propicien la no dependencia y el desarrollo autónomo de las investigaciones.

1 D os son las principales experiencias que han propiciado el tejido de esta red: El proyecto FOCYP (Formación de Públicos y $O$ fertas Culturales): investigación con equipos de trabajo en diez ciudades del país y coordinado por el Dr. Jorge G onzález; y el equipo de trabajo coordinado por el Dr. Jesús $G$ alindo, dedicado a capacitar equipos de investigadores en varias universidades del país y a dirigir múltiples proyectos de investigación.

Nuestra participación en el equipo de trabajo que construye la red está centrada en el diseño de software, en la capacitación y en la conformación de procedimientos automatizados para la creación de páginas en el WEB en el ámbito de las artes y la cultura en general. 
El uso del cómputo como herramienta imprescindible en el proceso de investigación es una realidad que no debe hacerse a un lado. Consideramos que desde la concepción de los proyectos, pasando por sus fases y resultados, debe tomarse en cuenta la disponibilidad económica de equipos y software, así como de una mentalidad que toma en cuenta estructuras de datos, organización de archivos y estructuras hipertextuales para conformar y desarrollar un proyecto de investigación.

En la medida en que se adopten estándares de uso general para la transferencia e intercambio de información (archivos ASCII, formatos GIF, JPEG y HTM) así como el uso de paquetes integrados para el manejo de procesadores de texto, diseño de gráficos, hoja electrónica y bases de datos, se podrán establecer lineamientos de compartibilidad e intercambio de información.

Estamos cada vez más convencidos de que el camino para usar al cómputo durante una investigación es a través de paquetes integrados en donde el usuario debe conocer su operación (evitando mayores dependencias con los desarrolladores o programadores) y puede automatizar algunas operaciones en su tratamiento (incrementando su cultura informática).

Es más importante poder trabajar, mostrar o difundir una aplicación en hoja electrónica (con recursos cada vez más poderosos) en cualquier equipo PC, que conocer e instalar temporalmente paquetes específicos que sólo estén disponibles en una o dos computadoras.

Desde luego que cuando las necesidades de una investigación impongan el uso de bases de datos o softwares de fuerte capacidad o muy especializados, debe considerarse el uso de equipo y software idóneo para dichas necesidades.

Respecto a la información compartible, son los formatos comunes de registro de información los que permitirán establecer criterios comunes entre los usuarios para actualizar, consultar, importar y exportar información.

La estructura operativa de la red considera solamente dos niveles de participación, el de coordinación que tiene la misión de coordinar las actividades de su nodo o equipo de trabajo y el nivel de los investigadores y creadores que son los que alimentan a la red con su trabajo.

El reto fundamental está en saber construir y participar en una red que tiende a una estructura horizontal en cuanto al suministro y acceso de información, con el propósito de transformar los conceptos de pertenencia y compartibilidad en los ámbitos del ciberespacio.

\section{ESFERA DE LA INVESTIGACIÓN}

Los elementos básicos que definen esta propuesta operativa son los siguientes:

1. Grupo de trabajo.

2. Proyecto de Investigación.

3. La Información. 


\section{InvestigacoónBiblictedóǵca v. 11 N o. 23 julio/ diciembre de 1997}

4. Herramientas de trabajo.

5. Medio de comunicación.

6. Nodo de la red.

Cada concepto implica una serie de definiciones, lineamientos básicos y procedimientos estándar que en su conjunto darían la forma operativa de la red.

\section{G rupo de trabajo}

El grupo de trabajo está conformado por un conjunto de coordinadores, investigadores y creadores que tienen una sede común o distribuida. Realizan uno 0 más proyectos y comparten recursos de cómputo.

El número de coordinadores depende de la organización del grupo y tienen la misión de cumplir funciones de organización y coordinación de proyectos y recursos. Junto con los investigadores y creadores, generan, intercambian y reinventan la información, sus contenidos y resultados.

Un ejemplo de sede común es el grupo formado por un diplomado, maestría 0 doctorado en una universidad. Aunque en oficinas, campus y hogares diferentes, los coordinadores e investigadores comparten un proyecto global común, además de participar en uno o más proyectos.

Ejemplos de grupos distribuidos son el proyecto FOCYP (subred dentro de la red) en donde coordinadores e investigadores tienen sus sedes en ciudades diferentes, todos con el mismo propósito de investigación; otro es el caso del grupo PASANDO EL SIGLO EN EL CINE (subred de FOCYP) en donde coordinadores y creadores tienen residencias en ciudades diferentes y crean una serie de televisión con un tema común. O tro grupo es el de la G eneración Macluhan, conformado por investigadores de la comunicación.

\section{El proyecto de investigación}

Los proyectos de la red pueden ser de muy diversos tipos, temas y propósitos, desde las investigaciones de un fenómeno social o cultural hasta proyectos lúdicos para conformar ciberespacios de creación.

En el marco de esta propuesta y como primer paso, los proyectos que se consideran son los relativos a las investigaciones sociales y a la cultura, con la perspectiva planteada en los diplomados y talleres ya realizados por miembros de la red en el ámbito universitario. Son proyectos que consideran una o más metodologías de investigación para tratar los temas y en casi todos los casos requieren del uso de herramientas de cómputo para su desarrollo y presentación.

El seguimiento y control de los proyectos en cada grupo se lleva a cabo con base en los lineamientos de los coordinadores y responsables de los proyectos. Como más adelante se presentará, la información relativa a ellos podrá ser mostrada en un apartado de la página en el Web, y por otro lado, en la parte de for- 
matos comunes, se presentará el estado actual del proyecto, tomando en cuenta los lineamientos que se describen a continuación.

La definición de las "fases de desarrollo" permitirá normar un criterio general para tener una visión de conjunto de uno o más proyectos, y facilitar la ubicación y estado actual de un proyecto y el tipo de información disponible y compartible con los miembros de la red. D esde un punto de vista macroscópico, consideramos el proyecto en cinco fases, que se llevan a cabo entre uno y tres años.

$>$ Fase de preparación.

> Fase de asentamiento.

> Fase del trabajo de campo.

> Fase de terminación.

$>$ Fase de difusión.

La fase de preparación corresponde al periodo de conformación del proyecto con base en los lineamientos establecidos por las coordinaciones respectivas. En el caso de un grupo universitario, corresponde al curso propedéutico. En otros casos, es el periodo para realizar el anteproyecto.

La fase de asentamiento principia con la fecha formal de inicio del proyecto y concluye cuando se han establecido claramente los objetivos, alcance y plan de trabajo de campo.

La fase del trabajo de campo generalmente es la más prolongada y responde a un plan de acción propio de cada proyecto.

La fase de teminación se puede considerar que inicia cuando el investigador ha terminado de recopilar la información de campo y sólo le queda asentarla, reflexionar y formular las conclusiones.

La fase de difusión es posterior a la edición del proyecto, ya sea en papel o en páginas del Web para su difusión. Su duración depende del peso y proyección de la investigación para mantener la atención de terceros en la red u otros medios.

\section{La información}

D el ilimitado universo de datos vinculados a una investigación, solamente circunscribimos aquella que será compartida en la red. Partimos de que toda la información digitalizable (texto, imagen y voz) que se proporcione a los espacios de consulta en la red, será compartible con todos los miembros de la red.

Para que la información sea eficientemente compartible debe tomar en cuenta "formatos comunes" para el registro de los datos, su almacenamiento y consulta en pantalla y en papel. En cuanto a los "formatos de registro de información básica", solamente enumeraremos los más importantes:

> Formato de registro de proyectos, con la información básica del investigador y del proyecto. D efinido por una tabla de hoja electrónica y consolidado 
más adelante en una base de datos que alimentará a la página correspondiente en el Web.

- Formato para el registro de la información del anteproyecto: contiene el planteamiento teórico, fundamentación, objetivos y alcance del proyecto. En una primera versión de este formato, se consideran los puntos planteados por el Mtro. Mariñes. Este formato y el anterior se emplearán en la fase de preparación de los proyectos.

> Formato para las refenencias bibliográficas, empleado a lo largo de todo el proyecto.

> Formato para las fases del proyecto: que permitirá conocer el estado actual del proyecto, y los resultados parciales a nivel macroscópico obtenidos a la fecha. Considera algunos aspectos tomados en cuenta en los formatos propios de cada grupo de trabajo para el seguimiento y control de cada proyecto.

- Formatos de consulta, que corresponden a la manera de presentar los resultados finales de los proyectos en la página del Web; se establecerá en su momento la estructura de los contenidos y los criterios de búsqueda (por temas).

\section{H erramientas de trabajo}

Consideramos al software y a los recursos de comunicación como las dos herramientas básicas de trabajo en la red.

Respecto al software proponemos las siguientes posibilidades en cuanto a lenguajes y estándares en tipos de archivos:

- Cualquier procesador de palabras que "exporte" los textos a archivos con formato ASCII; cualquier base de datos que exporte a formato DBF o tenga la posibilidad de exportar información de los campos a archivos ASCII con formatos en columnas o separados por comas; cualquier hoja electrónica que permita la "importación a EXCEL" o exporte información a formatos ASCII; cualquier generador de gráficos que permita generar archivos con formatos "gif o jpeg".

> Pensamos que el uso de un paquete como el OFFICE de Microsoft, que integra todas las aplicaciones anteriores (salvo los archivos para imágenes), es la opción más económica y de mayor alcance, principalmente en lo concerniente al uso de la hoja electrónica, que es una herramienta con mucho potencial para las investigaciones.

- No descartamos el uso de bases de datos más poderosas (SYBASE, ORACLE, INFO RMIX, etcétera), para manipular grandes cantidades de información, que exigen de mayor atención para su uso y administración, aspectos que visualizamos a mediano plazo.

- Para la integración de la información en el Web, proveniente de los softwares anteriores, el estándar por ahora será el HTML (V3.0). 
La transferencia de la información de los procesadores de palabras, bases de datos y hoja electrónica, al lenguaje empleado en las páginas del Web, puede hacerse con base en archivos puente en formato ASCII 0, por medio de programas de conversión directa de un formato particular al HTML.

La segunda herramienta de trabajo en la red la proporcionan los tres servicios básicos que ofrece INTERNET:

> El correo electrónico y las posibilidades que ofrece para enviar información auxiliar a través del "attach".

> Los foros de discusión.

$>$ El servicio de envío de archivos (ftp).

$>$ El servicio del World Wide Web.

Sugerimos que la manera de emplear y organizar el flujo de información por medio del correo electrónico sea abierta y dependa de la manera como cada miembro de la red desee establecer el contacto y comunicación con otros miembros ya conocidos 0 , por conocer.

La realización continua de foros de discusión es un componente vital para la intercomunicación "inteligente" de los miembros de la red, ya que se propiciará la mención directa a contenidos de información de las investigaciones de la red misma.

El uso del "ftp" permite enviar archivos medianos o grandes, situación que se presentará cuando inicie el flujo de imágenes o conjuntos de páginas en el Web, y que en su momento, requerirá de instructivos específicos. Los envíos de archivos (por "attach" o por "ftp") podrán ser empacados vía "zip".

Para participar en el ámbito de las comunicaciones en la red, el grupo de trabajo debe gestionar el acceso y espacio en disco requerido en un servidor, así como los servicios básicos de Internet (correo electrónico y envío de archivos de datos). Pueden existir tres casos:

$>$ Que el grupo de trabajo no tenga acceso a un Servidor de Internet.

> Q ue el grupo de trabajo sí tenga acceso a un Servidor de Internet.

$>$ Q ue el grupo de trabajo tenga un Servidor de Internet.

En el primer caso, que no es deseable y sí factible, el grupo en cuestión podrá copiar de un servidor de otro grupo de la red en operación, las páginas que considere pertinente e instalarlas localmente en un equipo de su grupo de trabajo, también deberá copiar en el servidor del grupo que le ofrezca apoyo, la información que desee compartir en el Web y actualizarla con la periodicidad que juzgue conveniente.

En el segundo caso, que será el más frecuente a corto y mediano plazos, los coordinadores del grupo de trabajo enviarán las actualizaciones de sus páginas al servidor disponible. Este servicio lo ofrecerá, generalmente, la instancia correspondiente en su universidad, o en algunos casos un servicio contratado en su ciudad. 


\section{Investigacón Biblictedóǵca v. 11 N o. 23 julio/ diciembre de 1997}

A mediano y largo plazos, consideramos que la mayoría de los grupos de trabajo tendrán su propio servidor (operando como subnodos de algún nodo de Internet en México) y podrán administrar y actualizar la información de sus páginas en la misma sede del grupo.

En cada caso, será necesario elaborar pequeñas guías para que los coordinadores informáticos de los grupos de trabajo, estructuren y actualicen las páginas correspondientes con criterios y lineamientos semejantes.

\section{El medio de comunicación}

Los servicios de Internet (correo electrónico, foros y ftp) y especialmente "las páginas" en el Web son propiamente "el medio" para presentar e intercambiar información entre los miembros de la red, y con otras redes y al público en general.

A través del Web la red podrá organizar la información de manera integral, estableciendo un espacio para su trabajo interno y otro para su interrelación con otras redes y en general, con la comunidad de usuarios del Web.

Proponemos que la "página de la red" contenga dos grandes componentes: el primero es el que mira hacia adentro, hacia la red misma y que operará como una INTRANET que sintetiza y presenta de manera ordenada, la información de interés a los coordinadores, investigadores y creadores de la red durante el desarrollo de su trabajo. Es el componente para el apoyo en el intercambio y desarrollo interno de las investigaciones en la red.

El segundo componente de la página en el Web es el que mira hacia afuera, para operar como una página en INTERNET que sintetiza y presenta de manera ordenada, la información que va a difundirse y es de interés para los coordinadores, investigadores y creadores de otras redes y latitudes.

El componente de INTRANET, a su vez, considera dos partes: la que toma en cuenta la información compartible de cada grupo de trabajo con base en los formatos comunes para el registro y consulta el estado de los proyectos, y la parte que permite la presentación y difusión abierta y libre de cada grupo de trabajo. El acceso a la información en ambas partes se da, en primera instancia por el grupo de trabajo, y en cada página de grupo, el acceso puede ser por el tipo de información: básica, del anteproyecto, bibliográfica y del estado actual de los proyectos.

El componente de INTERNET también presenta dos partes: la que describe los objetivos y propósitos de la red, sus estándares y procedimientos de comunicación y organización, así como la manera para que nuevos grupos se integren a la red. Por otro lado, considera la parte que permite la consulta a la información básica y resultados de los proyectos con base en una clasificación por temas 0 metodologías empleadas en los proyectos. 
Integración y comunicación en proyectos de investigación en el área... 79

\section{El N odo}

A manera de síntesis, un nodo de la red:

> Está conformado por un grupo de trabajo y vinculado a un conjunto de proyectos que tienen formatos de registro y contenidos de información compartible.

> Para conectarse de manera digital con otros nodos, los coordinadores, investigadores y creadores de un grupo de trabajo emplean el correo electrónico y el acceso a las páginas en el Web como medios para intercambiar y consultar información entre sí.

> La compartibilidad de la información entre los nodos se logra mediante el uso de estándares en los lenguajes empleados, formatos comunes para el registro de información básica, y bases de datos comunes dentro de una INTRANET de la red.

> Como parte de una integración general de la red, cada nodo considera lineamientos comunes de organización de la información para difundir e intercambiar los resultados de sus investigaciones y de las propuestas de creación, 0 criterios propios para mostrarla e intercambiarla de otras maneras en la red 0 con otras redes.

> La forma de establecer contacto entre los miembros de la red es mediante los foros o consultando las bases de datos con información estandarizada del registro de proyectos. Podría hacerse por dos vías: consultando las páginas de los grupos de trabajo en el componente de las páginas con formatos comunes en Intranet, o por el componente de consulta por temas o metodologías. En ambos casos se puede conocer la dirección electrónica del investigador e iniciar el contacto. Por medio de la consulta a la base de datos de la descripción de los anteproyectos del grupo, tendrá más elementos para analizar y estudiar los aspectos de interés y formular concretamente preguntas desde el primer contacto.

> La formulación de nuevos grupos de trabajo o proyectos de investigación puede tomar, como punto de partida, la síntesis de los resultados obtenidos al momento en la red, por otros grupos de trabajo o proyectos terminados.

Asumimos el carácter ambicioso de la red que queremos construir, y el gran reto que implica modificar, actualizar o inmiscuir a investigadores y creadores en los lenguajes y recursos informáticos de hoy en día. Sin embargo, el equipo humano de trabajo, que ya tiene varios años propugnando por un cambio de conciencia en el proceso de investigación en las ciencias sociales, está convencido de que sí es posible entrar al ciberespacio de manera constructiva y creativa, ya que tiene como banderas lo lúdico y las nuevas maneras de interaccionar con la realidad.

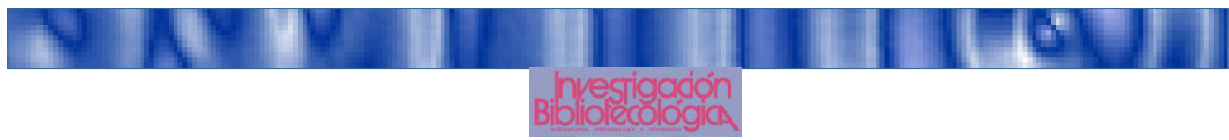

